\title{
Zero-Error Type of Chebyshev Polynomials
}

\author{
Lemin $\mathrm{Gu}$
}

\begin{abstract}
Zero-Error type of Chebyshev polynomials is one of Chebyshev polynomials extensions, it focus on "the zero error" and "the maximum absolute error minimization". Under the condition of $y(x)=0$, the existence question about Z-Chebyshev polynomials, which including the differential equations, the relevant definitions, the corresponding nature, the mathematical expressions, the recursive formula, the relationship between Z-Chebyshev polynomials and Chebyshev polynomials, are discussed and proved. Under the condition of $y(x) \neq 0$, the general features and characteristics about $\mathbf{Z}$ Chebyshev polynomials in the process of being realized, the algorithm to obtain this approximation solution, are introduced and given.
\end{abstract}

Index Terms-Chebyshev, polynomials, prediction, optimal approximation.

\section{QUESTIONS RAISED}

The optimal approximation elaboration originates from the machine design. In Chebyshev article "Involved Parallelogram Mechanical Principles" in 1857, he assumed the ideal design of the machine moved with $y(x)$ curvilinear motion, but the trajectory of the actual manufacturing of the machine movement path was $f(x)$, The optimal uniform approximation polynomials was the polynomials of the machines were designed to scale to achieve the minimum. Chebyshev proved a series of nature of optimal uniform approximation polynomials,introduced Chebyshev interleaved group and symbol discrimination law, and proved such polynomials was the optimal uniform approximation polynomials.

A change process cannot be no error, a big erroneous appearance often follows some kind of risk oncoming.Chebyshev optimal approximation to the maximum error of the change process is minimized, so as to minimize risk.

A process of change exists the beginning point to the end point, whether doesn't have any error to start accurately from the beginning, also does not have any error to arrive the end point accurately, this is an important topic on the optimal approximation. However we will find, because in Chebyshev polynomials the beginning and the end point error all is maximum, this makes us unable to start from the beginning point, also unable to reach the terminal point. So when takes the beginning point and end point as a ideal point of "no-error" or "zero error" to deal with, needs to carry on the promotion to Chebyshev optimal approximation principle.

For promotion of Chebyshev polynomials, [1] gave two

Manuscript received April 12, 2013; revised June 29, 2013.

Lemin $\mathrm{Gu}$ is with the Tongji University, China (e-mail: gulemin@tongji.edu.cn). new classes of Chebyshev polynomials and called the third, the fourth kind of Chebyshev polynomials; [2] provided predictive type of Chebyshev polynomials; [3] studied on the best uniform approximation polynomial of Cosine Function type; [4] discussed nonlinear Chebyshev solution realization question; [5] gave some application in the calculation of absolute and relative methods.

Chebyshev polynomials $T_{n}(x)=\cos (n \arccos x)$ defined in the interval $[-1,1]$ can use the trigonometric functions to express the orthogonal polynomials simply. Taking $n=2,3,4,5$, drawing $T_{2}(x) \sim T_{5}(x)$ in Fig.1, in which blue lines are Chebyshev polynomials, T2, T3, T4, T5 express $T_{2}(x), T_{3}(x), T_{4}(x), T_{5}(x) \quad$ separately. By $\quad$ Fig. 1 $T_{n}(x)$ curve draws out some questions as follows.

1) $T_{n}(x)$ curve in the starting endpoint -1 is the maximum absolute value of error, so it is unsuitable a small amount of change description in the initial stage. For example of chemical reaction, in its initial stage, measures are generally very weak, the error is larger than its amount of change cannot be explained;

2) $T_{n}(x)$ at the end point 1 is the maximum error, if we want that the error of the target point is as small as possible, or even no error, then $T_{n}(x)$ polynomials is given exactly precisely the opposite result, at the end the error will be the largest.

3) $T_{n}(x)$ at the endpoint -1 and 1 are the maximum or minimum value errors, but not extreme points, so the curve will not turn round. The change of curve outside $[-1,1]$ continues by the monotone increasing way, this will cause with the extrapolation method establishment forecast error, the erroneous value to be bigger than the endpoint error, namely will be bigger than the biggest absolute value error in the sector $[-1,1]$.

4) Therefore may believe that $T_{n}(x)$ polynomials is suitable generally for the description which changes in the sector $(a, b)$, but not suitable for decrypting the situation of zero-error endpoints, also not suitable for forecasting outside the sector $[a, b]$.

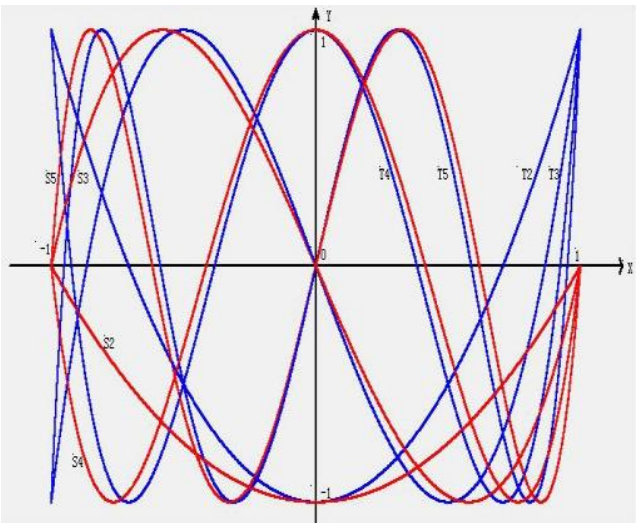

Fig. 1. The curves of $T_{2}(x) \sim T_{5}(x)$ and $S_{2}(x) \sim S_{5}(x)$ in [-1,1] 
5) If the tentative plan has one kind with Chebyshev polynomials similar multinomial, this possibility? Look at Figure 1, the red curve of S2, S3, S4, S5, there are both the same and different between the $S_{n}(x)$ curve and $T_{n}(x)$ curve.

The so-called same place includes: (1) the sectors $[-1,1]$ is the same; (2) the maximum and minimum value is the same, the maximum value is 1 , the minimum value is -1 ;(3) the staggered nature is the same, the $S_{n}(x)$ curve extreme point also appear in the positive and negative interaction form.

The so-called different place includes: the $S_{n}(x)$ at the endpoint of the curve -1 and 1 is zero, in the article is called "the zero-error" point, is exactly opposite with the $T_{n}(x)$ curve, is a process from the zero-error beginning to the zero-error ending.

6) Looked from the $S_{n}(x)$ curve change, if with the extrapolated method establishment forecast, may infer outside point 1 has a sector, in this sector forecast error could not be bigger than the most greatly absolute error, thus will cause the prediction to become knowable and controllable.

Such idea source in one kind of supposition:

1) the beginning of change is the zero-error point;

2) The process is according to the maximum absolute deviation minimized;

3) The goal achieved point is the ideal zero-error point;

4) The prediction is possible within a certain range.

It is well known, Chebyshev polynomials form is only, but Fig. 1 comes out actually the new similar Chebyshev polynomials graph, then Chebyshev polynomials whether also only? How is the new curve a mathematics type, what main characteristic does it have? What does relation have with Chebyshev polynomials? Questions like these will be following.

It is necessary to explore the existence of the $S_{n}(x)$ polynomials, to discuss its realization, and to study this kind of optimal approximation issues, its theory and the practical value may be certain existence.

\section{The EXISTEnCE Question ABOUt Zero-ERror TyPE OF CHEBYSHEV POLYNOMIALS}

Definition 1 Definition differential equation

$$
\left[1-\left(x \cos \frac{\pi}{2 n}\right)^{2}\right] S_{n}^{\prime \prime}(x)-\cos ^{2} \frac{\pi}{2 n} x S_{n}^{\prime}(x)+n^{2} \cos ^{2} \frac{\pi}{2 n} S_{n}(x)=0
$$

The solutions to this equation are called Chebyshev's zero-error type polynomials, simply expressed as ZChebyshev polynomials and indicated with symbol $S_{n}(x)$.

$$
S_{n}(x)=\cos \left[n \arccos \left(x \cos \frac{\pi}{2 n}\right)\right] \quad(-1 \leq x \leq 1, n=2,3, \ldots)
$$

Proof: By setting $z=x \cos \frac{\pi}{2 n}, \theta=\arccos z$ to (2), two derivatives of $S_{n}(x)=\cos n \boldsymbol{\theta}$ respectively are:

$$
\begin{aligned}
& S_{n}^{\prime}(x)=\frac{d S_{n}(x)}{d x}=\frac{d S_{n}(x)}{d \theta} \frac{d \theta}{d z} \frac{d z}{d x}=n \frac{\sin n \theta}{\sin \theta} \cos \frac{\pi}{2 n} \\
& S_{n}^{\prime \prime}(x)=n \frac{x \sin n \theta}{\sin ^{3} \theta} \cos ^{3} \frac{\pi}{2 n}-n^{2} \frac{\cos n \theta}{\sin ^{2} \theta} \cos ^{2} \frac{\pi}{2 n}
\end{aligned}
$$

Substitute (1) and note $z=\cos \theta, 1-z^{2}=\sin ^{2} \theta$, the equation is established.

Definition 2 In the interval $[-1,1]$, setting $z=x \cos \frac{\pi}{2 n}$, $(n=2,3, \ldots)$ when $n \geq 2$, the $S_{n}(x)$ polynomials satisfy the recurrence relation:

$$
\left\{\begin{array}{l}
S_{0}(z)=1, \quad S_{1}(z)=z \\
S_{n}(z)=2 z S_{n-1}(z)-S_{n-2}(z) \quad(n=2,3, \ldots)
\end{array}\right.
$$

Proof: by the Definition 1 of recursive relationship, $S_{n}(x)$ is starting from $n=2$. Taking $n=2$, by (3)

$$
S_{2}=2 z S_{1}-S_{0}=2 z^{2}-1=2\left(x \cos \frac{\pi}{4}\right)^{2}-1=x^{2}-1,
$$

is the same with (2)

$$
S_{2}(x)=\cos (2 \arccos z)=2 \cos ^{2}(\arccos z)-1=2 z^{2}-1=x^{2}-1
$$

When $n=3$, by (2) or (3) has

$$
S_{3}=2 z S_{2}-S_{1}=4 \cos ^{3} \frac{\pi}{6} x^{3}-3 \cos \frac{\pi}{6} x=\frac{3}{2} \sqrt{3} x^{3}-\frac{3}{2} \sqrt{3} x
$$

The first $5 S_{n}(x)$ polynomials expressed with the trigonometrically function are:

$$
\begin{aligned}
& S_{2}(x)=2 \cos ^{2} \frac{\pi}{4} x^{2}-1 \\
& S_{3}(x)=4 \cos ^{3} \frac{\pi}{6} x^{3}-3 \cos \frac{\pi}{6} x \\
& S_{4}(x)=8 \cos ^{4} \frac{\pi}{8} x^{4}-8 \cos ^{2} \frac{\pi}{8} x^{2}+1 \\
& S_{5}(x)=16 \cos ^{5} \frac{\pi}{10} x^{5}-20 \cos ^{3} \frac{\pi}{10} x^{3}+5 \cos \frac{\pi}{10} x
\end{aligned}
$$

or expressed with the algebra polynomials:

$$
\begin{aligned}
& S_{2}(x)=x^{2}-1 \\
& S_{3}(x)=\frac{3}{2} \sqrt{3} x^{3}-\frac{3}{2} \sqrt{3} x \\
& S_{4}(x)=(3+2 \sqrt{2}) x^{4}-2(2+\sqrt{2}) x^{2}+1 \\
& S_{5}(x)=\frac{5}{4} \sqrt{50+22 \sqrt{5}} x^{5}-\frac{5}{2} \sqrt{25+10 \sqrt{5}} x^{3}+\frac{5}{4} \sqrt{10+2 \sqrt{5}} x
\end{aligned}
$$

The graphs of these polynomials (up to $n=5$ ) are shown in Fig. 1 (red line), and they denoted symbols $S n=S_{n}(x)$.

By the above, main properties of $S_{n}(x)$ polynomials are introduced as follows.

Nature 1 (zero point) When $n=2,3, \ldots$, in the interval $[-1,1] S_{n}(x)$ polynomials have $n$ different zero points.

$$
x_{k}=\frac{\cos \frac{2 k-1}{2 n} \pi}{\cos \frac{\pi}{2 n}} \quad(k=1,2, \ldots, n \quad n \geq 2)
$$


Nature 2 (extreme point) When $n=2,3, \ldots$, in the interval $[-1,1] \quad S_{n}(x)$ polynomials have $n-1$ different extremum points.

$$
x_{j}=\frac{\cos \frac{j}{n} \pi}{\cos \frac{\pi}{2 n}} \quad(j=1,2, \ldots, n-1 \quad n \geq 2)
$$

in turn make $S_{n}(x)$ polynomials obtain the maximum value 1 and minimum value -1 .

Nature 3 (coefficient) In the interval $[-1,1]$, the most high-order coefficient of $S_{n}(x)$ polynomials is:

$$
a_{n}=2^{n-1} \cos ^{n} \frac{\pi}{2 n} \quad(n=2,3, \ldots)
$$

Nature 4 (first coefficient is 1 polynomials, error expression) In the interval $[-1,1]$, when first coefficient is $1, S_{n}(x)$ polynomials become $s_{n}(x)$ polynomials that indicated with lowercase letters. The relations of $S_{n}(x)$ and $s_{n}(x)$, as well as the relations $s_{n}(x)$ and zero deviation value $E_{n}$ can be described:

$$
S_{n}(x)=\frac{1}{E_{n}} s_{n}(x) \quad E_{n}=1 / 2^{n-1} \cos ^{n} \frac{\pi}{2 n}
$$

when $n=2,3,4,5$ :

$$
\begin{array}{ll}
s_{2}(x)=x^{2}-1 & S_{2}(x)=\left(2^{1} \cos ^{2} \frac{\pi}{4}\right) s_{2}(x) \\
s_{3}(x)=x^{3}-x & S_{3}(x)=\left(2^{2} \cos ^{3} \frac{\pi}{6}\right) s_{3}(x) \\
s_{4}(x)=x^{4}-2(2-\sqrt{2}) x^{2}+3-2 \sqrt{2} & S_{4}(x)=\left(2^{3} \cos ^{4} \frac{\pi}{8}\right) s_{4}(x) \\
s_{5}(x)=x^{5}-\frac{5-\sqrt{5}}{2} x^{3}+\frac{3-\sqrt{5}}{2} x & S_{5}(x)=\left(2^{4} \cos ^{5} \frac{\pi}{10}\right) s_{5}(x)
\end{array}
$$

The $s_{n}(x)$ polynomials may analogize when $n \geq 6$.

Note that with a subscript $s_{n}(x)$ and later $s(x)$ is two different functions.

\section{REALIZATION OF Z-CHEBYSHEV OPTIMAL APPROXIMATION}

\section{A. Overview}

Find a function $s(x)$ in the selected class functions, lets it be known function $y(x)$ in a certain sense of approximation, extracts the error that $s(x)$ expressed approximately $y(x)$ produces, this is the approximation of function question. The relations of $s(x)$ and $S_{n}(x)$ are, when $y(x)=0$ then $s(x)=S_{n}(x)$, at this time the $y(x)=0$ means a horizontal straight line. When $y(x) \neq 0$, the $s(x)$ polynomials realization question will be drawn out.

\section{B. Z-Chebyshev Optimal Approximation Question}

Theorem 1 Set function $y(x), s(x)$ in the interval $[a, b]$ smooth, continuous and derivable, $s(x)=s(x, \boldsymbol{a})$ can be expressed as $s(x, \boldsymbol{a})=a_{0} \varphi_{0}(x)+a_{1} \varphi_{1}(x)+\ldots+a_{n} \varphi_{n}(x)=\sum_{j=0}^{n} a_{j} \varphi_{j}(x)$

where $\varphi_{0}, \varphi_{1}, \cdots, \varphi_{n}$ in $[a, b]$ linear independence, or $s(x)=s(x, \boldsymbol{a})$ given by the algebra polynomials

$$
s(x, \boldsymbol{a})=a_{0}+a_{1} x+\ldots+a_{n} x^{n}=\sum_{j=0}^{n} a_{j} x^{n}
$$

where parameter $\boldsymbol{a}=\left(a_{0}, a_{1}, \ldots, a_{n}\right)$ is the any real number. In order to enable $s(x)$ to approach $y(x)$, when the error function $r(x)=r(x, \boldsymbol{a})=y(x)-s(x, \boldsymbol{a})$ satisfies the following relations:

$$
\left\{\begin{array}{l}
r\left(x, \boldsymbol{a}^{*}\right)=y(x)-s\left(x, \boldsymbol{a}^{*}\right)=0 \\
E^{*}=\max _{a \leq x \leq b}\left|r\left(x, \boldsymbol{a}^{*}\right)\right| \max _{a \leq x \leq b}\left|y(x)-s\left(x, \boldsymbol{a}^{*}\right)\right|=\min \\
r\left(x, \boldsymbol{a}^{*}\right)=y(x)-s\left(x, \boldsymbol{a}^{*}\right)=0
\end{array}\right.
$$

This is Chebyshev's zero error type optimal approximation question, simply expressed as Z-Chebyshev optimal approximation question.

Theorem 2 The $s(x)$ is the $y(x)$ Z-Chebyshev polynomials full essential condition is that the $s(x)$ at the end of $[a, b]$ have 2 zero-error points, in $(a, b)$ there are at least $n$ turns into "positive" and "negative" phase deviation points, altogether have $n+2$ points $a=x_{1}<x_{2}<\ldots<x_{n+2}=b$, make

$$
\left\{\begin{array}{l}
y\left(x_{1}\right)-s\left(x_{1}, \boldsymbol{a}^{*}\right)=0, \\
y\left(x_{j}\right)-s\left(x_{j}, \boldsymbol{a}^{*}\right)=\sigma(-1)^{j} E^{*} \quad(j=2, \ldots, n+1) \\
y\left(x_{n+2}\right)-s\left(x_{n+2}, \boldsymbol{a}^{*}\right)=0
\end{array}\right.
$$

where $\sigma= \pm 1$ decided by $y(x)$ and $n$.Called $E^{*}(>0)$ is the optimal approximation error value; Called $\boldsymbol{a}^{*}=\left(a_{0}^{*}, a_{1}^{*}, \ldots, a_{n}^{*}\right)$ the optimal approximation parameter; Called $s\left(x, \boldsymbol{a}^{*}\right) \mathrm{Z}$ Chebyshev optimal approximation polynomials.

\section{Specific Algorithms (5 steps below):}

Step 1. Chooses $n$ points $x_{1}^{(0)}, x_{2}^{(0)}, \ldots, x_{n}^{(0)}, x_{n+1}^{(0)}, x_{n+2}^{(0)}$ as a group of approximate Chebyshev staggered points in $[a, b]$, and the two endpoints $x_{1}=a, x_{n+2}=b$ as the zero error points.

Step 2. By the series of linear equation of $n+2$ equation constitution, can obtain the initial solution: the parameter $\boldsymbol{a}=\left(a_{0}, a_{1}, \ldots, a_{n}\right)$ and $\varepsilon$.

$$
\begin{gathered}
\left\{\begin{array}{l}
s\left(x_{1}^{(0)}\right)=y\left(x_{1}^{(0)}\right) \\
s\left(x_{j}^{(0)}\right)-(-1)^{j} \varepsilon=y\left(x_{j}^{(0)}\right) \quad j=2,3, \ldots, n+1 \\
s\left(x_{n+2}^{(0)}\right)=y\left(x_{n+2}^{(0)}\right)
\end{array}\right. \\
\Leftrightarrow\left\{\begin{array}{l}
a_{0}+a_{1} x_{1}^{(0)}+\ldots+a_{n}\left(x_{1}^{(0)}\right)^{n}=y\left(x_{1}^{(0)}\right) \\
a_{0}+a_{1} x_{2}^{(0)}+\ldots+a_{n}\left(x_{2}^{(0)}\right)^{n}-\varepsilon=y\left(x_{2}^{(0)}\right) \\
\ldots \ldots \\
a_{0}+a_{1} x_{n+1}^{(0)}+\ldots+a_{n}\left(x_{n+1}^{(0)}\right)^{n}+(-1)^{n+1} \varepsilon=y\left(x_{n+1}^{(0)}\right) \\
a_{0}+a_{1} x_{n+2}^{(0)}+\ldots+a_{n}\left(x_{n+2}^{(0)}\right)^{n}=y\left(x_{n+2}^{(0)}\right)
\end{array}\right.
\end{gathered}
$$


Step 3. Through a linear equations that constituted by $n$ derivative-equation can obtain the solution: the $y(x)-s(x)$ approximate extreme points, recorded as $\left\{x_{j}^{(1)}\right\}$.

$$
\begin{aligned}
& y^{\prime}\left(x_{j}^{(0)}\right)-s^{\prime}\left(x_{j}^{(0)}\right)=0, \quad j=2,3, \ldots, \quad n+1 \\
& \Leftrightarrow\left\{\begin{array}{l}
y^{\prime}\left(x_{2}^{(0)}\right)-s^{\prime}\left(x_{2}^{(0)}\right)=0 \\
y^{\prime}\left(x_{3}^{(0)}\right)-s^{\prime}\left(x_{3}^{(0)}\right)=0 \\
\ldots \ldots \\
y^{\prime}\left(x_{n+1}^{(0)}\right)-s^{\prime}\left(x_{n+1}^{(0)}\right)=0
\end{array}\right.
\end{aligned}
$$

Step 4. Replace $\left\{x_{j}^{(0)}\right\}$ with $\left\{x_{j}^{(1)}\right\}$, transfer step 2, or replace $\left\{x_{j}^{(k)}\right\}$ with $\left\{x_{j}^{(k+1)}\right\},(k=0,1,2, \ldots)$ transfer step 2 .

Step 5. Duplicate above computation, until parameter iterative error $\boldsymbol{a}^{(k+1)}-\boldsymbol{a}^{(k)} \quad(k=0,1,2, \ldots$ iterative times $)$ meet the precision requirements, can prove $\boldsymbol{a}^{(k+1)} \rightarrow \boldsymbol{a}^{*},|\varepsilon| \rightarrow E^{*}$ when $k \rightarrow \infty$.

\section{Example}

Using descending powers methods, in the interval $[0,1]$, the function $y(x)=e^{x}$ expand into the $s(x)$ quadratic polynomials, and compare with the Taylor Expansion.

As we all know, the direct application of Chebyshev polynomials, in function Taylor launches the number of terms saves, for this reason also carries on with the $s(x)$ polynomials.

1) A descending powers: In the interval $[0,1]$, the Taylor's cubic polynomial is

$$
P_{3}(x)=1+x+\frac{1}{2} x^{2}+\frac{1}{6} x^{3},
$$

the cubic polynomial $s_{n}(x)$ is

$$
s_{3}(x)=x^{3}-\frac{3}{2} x^{2}+\frac{1}{2} x
$$

eliminates $x^{3}$ item, can get the quadratic polynomial

$$
s(x)=P_{3}(x)-\frac{1}{6} s_{3}(x)=1+\frac{11}{12} x+\frac{3}{4} x^{2}
$$

in which the maximum interruption errors

$$
\boldsymbol{\varepsilon}_{\max }=e^{x}-\left.\left(1+\frac{11}{12} x+\frac{3}{4} x^{2}\right)\right|_{x=1}=0.0516
$$

it is same with the Taylor's cubic polynomial.

2) Two descending powers: Takes the Taylor's quartic polynomial

$$
P_{4}(x)=1+x+\frac{1}{2} x^{2}+\frac{1}{6} x^{3}+\frac{1}{24} x^{4}
$$

In the interval $[0,1]$, the quartic polynomial $s_{n}(x)$ is

$$
s_{4}(x)=x^{4}-2 x^{3}+\frac{\sqrt{2}+1}{2} x^{2}-\frac{\sqrt{2}-1}{2} x
$$

eliminates $x^{4}$ item, again eliminates $x^{3}$ item, can get the quadratic polynomial

$$
s(x)=1+\frac{41+\sqrt{2}}{48} x+\frac{41-\sqrt{2}}{48} x^{2}
$$

which the maximum error is $\varepsilon_{\max }=0.0118$ at the point of $x=0.219$.

3) Repeatedly descending powers, can get the ZChebyshev optimal approximation quadratic polynomial

$$
s\left(x, a^{*}\right)=1+0.8708 x+0.84748 x^{2}
$$

the maximum errors $E^{*}=0.0135$ appears in the $x_{1}=0.2254$ and $x_{2}=0.8023$. This is ensuring ZChebyshev optimal approximation under the endpoint $x=0$ and $x=1$ no-error condition, makes the quadratic polynomial obtains the absolute maximum error minimizing in the sector $[0,1]$.

4) Prediction: Using the extrapolation method, supposes $\Delta x$ for the controllable biggest prediction sector, $E^{*}$ for the knowable biggest prediction error. When takes $\Delta x=0.07$, the prediction error is

$$
\varepsilon(1+\Delta x)=\varepsilon(1.07)=e^{1.07}-s(1.07)=0.0133<E^{*}
$$

It can be seen that within about $7 \%$ outside $[0,1]$, the prediction is knowable and controllable.

\section{Curve Fitting Method}

\section{A. Overview}

When $f(x, \boldsymbol{a})$ is the parameter $\boldsymbol{a}=\left(a_{0}, a_{1}, \ldots, a_{n}\right)$ linear function fitting problem is called as "the linear function class fitting problem", such as $f(x)$ is polynomial equation. When $f(x, \boldsymbol{a})$ is the parameter $\boldsymbol{a}$ nonlinear function fitting problem as "the nonlinear function class fitting problem ", such as $f(x)$ is Logistic equation and MMF equation, etc. When kneading them together, forming a general function class of fitting, and is no longer distinguished between linear and nonlinear.

\section{B. No solution or Unusual Solutions}

In this paper what to discuss Z-Minimax curve fitting is the ideal or more ideal conditions, but actually is far from complex. For discrete data obtained from scientific experiments, have greater randomness and discreteness, as well as measurement error, Chebyshev on the parameters $\boldsymbol{a}=\left(a_{0}, a_{1}, \ldots, a_{n}\right)$ solution may not be set up.

For instance, non-interleaved group Chebyshev solution is an unusual solution, the maximum or minimum error is a non positive and negative stagger, but the approximation solution still exists, this often may appear in nonlinear function class optimal approximation.

To simplify the discussion, the article special provisions that about the parameter $\boldsymbol{a}$ solution is the existence, do not expand on the discussion on non-solutions or unusual solutions, for this type of problem is more complex, to be in another paper commenced.

\section{Z-Minimax Curve Fitting Method}

Definition 3 For a given $m$ discrete set of data 
$\left(x_{i}, y_{i}\right) i=1,2, \ldots, m$, takes curve fitting equation $y=f(x, \boldsymbol{a})$, the parameter $\boldsymbol{a}=\left(a_{0}, a_{1}, \ldots, a_{n}\right),(n \leq m-2)$ selected according to the criterion that the endpoint is the zero error and the absolute maximum deviation is minimal, namely satisfies the objective function:

$$
\left\{\begin{array}{l}
y_{j}-f\left(x_{j}, \boldsymbol{a}\right)=0 \quad j=1, m \\
E=E(\boldsymbol{a})=\max _{1 \leq i \leq m}\left|r_{i}\right|=\max _{1 \leq i \leq m}\left|y_{i}-f\left(x_{i}, \boldsymbol{a}\right)\right|=\min
\end{array}\right.
$$

Under this criterion constitutes the curve-fitting method is called Zero-error Minimax curve fitting method, or ZMinimax curve fitting method in short.

Theorem 3 By Definition 3, if there exits the function solution $\boldsymbol{a}=\boldsymbol{a}^{*}$, causes the fitting criterion (17) establishment, then the unique characterization of the fitting equation $f\left(x, \boldsymbol{a}^{*}\right)$ is that there are two zero-error point $x_{1}, x_{m}$ and at least $n$ points $x_{1 i}, x_{2 i}, \ldots, x_{n i}$,in which $x_{1 i}<x_{2 i}<\ldots<x_{n i}, 1<i<m$, cause

$$
\left\{\begin{aligned}
y_{i}-f\left(x_{i}, \boldsymbol{a}^{*}\right) & =0 & (i=1, m, \quad i \neq j) \\
y_{j}-f\left(x_{j}, \boldsymbol{a}^{*}\right) & =\sigma(-1)^{k} E^{*} & \\
(j & =1 i, 2 i, \ldots, n i, & 1<i<m, \quad k=1,2, \ldots)
\end{aligned}\right.
$$

where $\sigma= \pm 1$, decided by $f(x)$ and $n \cdot j=1 i, 2 i, \ldots, n i$ means $n$ points taken in any of $1<i<m$ points. Called $\boldsymbol{a}^{*}$ "optimal parameters of Z-minimax approximation", referred to as “*”, simply called "optimal parameters". If the equation set by (18) fail to establish, the (17) minimization fail either, and the Theorem 3 solution is not a valid solution.

\section{Mathematical Model}

Richards model was in 1959 Richards in the von Bertalanffy growth model based on the generalized treatment of new growth model with 4 parameters, each parameter's reasonableness is very important in the biology [6], [7]. To simplify the expression, here represented by $\boldsymbol{a}=\left(a_{0}, a_{1}, a_{2}, a_{3}\right)$ four parameters:

$$
f(x)=\frac{a_{0}}{\left(1+a_{1} e^{-a_{2} x}\right)^{1 / a_{3}}} \quad \text { or } \quad f(x)=\frac{a_{0}}{\left|1+a_{1} e^{-a_{2} x}\right|^{1 / a_{3}}}
$$

\section{E. Curve Fitting Error}

For curve fitting, the commonly used curve fitting error expresses the curve fitting result fit and unfit quality, also is called "mean absolute percentage error", defined as:

$$
\text { Mape }=\frac{1}{m} \sum_{i=1}^{m}\left|\frac{r_{i}}{y_{i}}\right| \times 100 \%=\frac{1}{m} \sum_{i=1}^{m}\left|\frac{y_{i}-f\left(x_{i}\right)}{y_{i}}\right| \times 100 \%
$$

\section{The Application in Census, the 4TH to THE 6TH PHASE IN CHINA}

\section{A. Overview}

In the world economic adjustment transformation, almost all the countries take the census work seriously, the development of population census become internationally eye focus. The census provides a clearer picture of the population structure, quality, helps stabilizing economy development in a long run. Also, it promotes the

\begin{tabular}{|c|c|c|c|c|c|c|c|c|c|c|c|}
\hline \multirow[b]{2}{*}{$i$} & \multicolumn{2}{|c|}{ Actual value } & \multicolumn{3}{|c|}{ Fitting value } & \multirow[b]{2}{*}{$i$} & \multicolumn{2}{|c|}{ Actual value } & \multicolumn{3}{|c|}{ Fitting value } \\
\hline & $\begin{array}{c}x_{i} \\
\text { (year) }\end{array}$ & $\begin{array}{c}y_{i} \\
\text { (million) }\end{array}$ & $\begin{array}{c}f\left(x_{i}\right) \\
\text { (million) }\end{array}$ & $\begin{array}{c}r_{i} \\
\text { (millio }\end{array}$ & $\begin{array}{c}R_{i} \\
\quad(\%) \\
\end{array}$ & & $\begin{array}{c}x_{i} \\
\text { (year) }\end{array}$ & $\begin{array}{c}y_{i} \\
\text { (million) }\end{array}$ & $\begin{array}{c}f\left(x_{i}\right) \\
\text { (million) }\end{array}$ & $\begin{array}{c}r_{i} \\
\text { (million) }\end{array}$ & $\begin{array}{l}R_{i} \\
(\%)\end{array}$ \\
\hline 1 & 1990 & 1143.33 & 1143.33 & 0 & 0 & 1 & 2000 & $1267^{\prime} 43$ & 1267.43 & 0 & 0 \\
\hline 2 & 1991 & 1158.23 & 1157.70 & 0.53 & 0.046 & 2 & 2001 & $1276^{\prime} 27$ & 1276.12 & 0.15 & 0.012 \\
\hline 3 & 1992 & 1171.71 & 1171.78 & -0.07 & -0.006 & 3 & 2002 & $1284 ' 53$ & 1284.42 & 0.11 & 0.009 \\
\hline 4 & 1993 & 1185.17 & 1185.52 & -0.35 & -0.030 & 4 & 2003 & $1292 ’ 27$ & 1292.38 & -0.11 & -0.009 \\
\hline 5 & 1994 & 1198.50 & 1198.86 & -0.36 & -0.030 & 5 & 2004 & $1299^{\prime} 88$ & 1300.03 & -0.15 & -0.012 \\
\hline 6 & 1995 & 1211.21 & 1211.74 & -0.53 & -0.044 & 6 & 2005 & $1307^{\prime} 56$ & 1307.41 & 0.15 & 0.012 \\
\hline 7 & 1996 & 1223.89 & 1224.11 & -0.22 & -0.018 & 7 & 2006 & $1314^{\prime} 48$ & 1314.53 & -0.05 & -0.004 \\
\hline 8 & 1997 & 1236.26 & 1235.90 & 0.36 & 0.029 & 8 & 2007 & $1321 ' 29$ & 1321.42 & -0.13 & -0.010 \\
\hline 9 & 1998 & 1247.61 & 1247.08 & 0.53 & 0.043 & 9 & 2008 & $1328^{\prime} 02$ & 1328.11 & -0.09 & -0.006 \\
\hline 10 & 1999 & 1257.86 & 1257.60 & 0.26 & 0.021 & 10 & 2009 & $1334^{\prime} 50$ & 1334.60 & -0.10 & -0.007 \\
\hline 11 & 2000 & 1267.43 & 1267.43 & 0 & 0 & 11 & 2010 & $1340 ’ 91$ & 1340.91 & 0 & 0 \\
\hline \multicolumn{3}{|c|}{ Results } & \multicolumn{3}{|c|}{$\begin{array}{l}\max \left|r_{i}\right|=0.53 \\
\max \left|R_{i}\right|=0.046 \% \\
\text { Mape }=0.024 \%\end{array}$} & \multicolumn{3}{|c|}{ Results } & \multicolumn{3}{|c|}{$\begin{array}{l}\max \left|r_{i}\right|=0.15 \\
\max \left|R_{i}\right|=0.012 \% \\
\text { Mape }=0.007 \%\end{array}$} \\
\hline \multicolumn{3}{|c|}{ The equation } & \multicolumn{3}{|c|}{ Formula (21) } & \multicolumn{3}{|c|}{ The equation } & \multicolumn{3}{|c|}{ Formula (22) } \\
\hline
\end{tabular}
comprehensive developmen has a very important significance.

TABLE I: ZERO-ERROR OPTIMAL APPROXIMATION TO 1990-2000 AND 2000-2010 CHINESE POPULATION CHANGE DATA PROCESSING

Using curve fitting method to processing population data may find the population change certain rules, and will forecast the future population development possible tendency and quantity. The census once every 10 years, due to a high degree of attention, the data obtained with certain accuracy. The census year population data as ideal for zeroerror data, the variation of the population between the two census years, uses the curve fitting method to explore. The Z-Chebyshev optimal approximation criterion is a good criterion in many curve fitting criterions. It will have a maximum error minimization throughout the process, and give a knowable and controllable prediction for the future change. In the analysis of results, as long as it clear maximum rationality, then in this section of scope changes the rationality of rule may easily be established.

According to the National Bureau of the China Statistical Yearbook 1990-2000 and 2000-2010 population of China (excluding Hong Kong, Macau, Taiwan) $11 \times 2$ sets of data are shown in TABLE 1 left [8], in which 4th 1990, 5th 2000, 6th 2010 are census year, so the data might think do not 
have any deviation and the accurate value data.

In the Table I $x_{i}$ is the year; $y_{i}$ is the population actual value (million); $f\left(x_{i}\right)$ is the fitting value (million); $r_{i}$ is the absolute error (million); $R_{i}$ is the relative error (\%).

\section{B. Data Processing}

Curve fitting method given by equation (18) and (19), to the 1990-2000 group data Richards equation is:

$$
f\left(x_{i}\right)=\frac{1351.743}{\left(1+5.4373 e^{-0.159094\left(x_{i}-1989\right)}\right)^{1 / 10.3282}}
$$

The equation of data processing results are shown in Table I and some conclusions as follows:

1) Minimize the maximum absolute error $E^{*}=\max \left|r_{i}\right|=0.53 \quad$ (million), the Maximum relative error $\max \left|R_{i}\right|=0.046 \%$, and the curve fitting error Mape $=0.024 \%$;

2) In 1990 and 2000 is two census years, the data accuracy is very high, so the endpoints is zero error points, also consistent with the facts.

3) Using the Richards equation to data processing has a certain degree of accuracy.

Using the same way on processing 2000-2010 group data, gets Richards equation with absolute value:

$$
f\left(x_{i}\right)=\frac{1416.2605}{\left|1-1.291444 e^{0.017496\left(x_{i}-1999\right)}\right|^{-1 / 10.426185}}
$$

Results are shown in Table I right side. Compares with the first group of data, several conclusions are explained as follows:

1) Minimize the maximum absolute error $E^{*}=\max \left|r_{i}\right|=0.15$ (millions), the Maximum relative error $\max \left|R_{i}\right|=0.012 \%$ and the curve fitting error Mape $=0.007 \%$;

2) Compares with the 1 st group of data processing results, all surpasses the 1st group. For a large population of 1.3 billion, the fitted maximum absolute error is only 0.15 million, and the result is ideal. This indicates Richards equation regarding the Chinese population change description, has the very high accuracy.

3) From the Richards equation of ultimate meaning, if in accordance with the current trend of development, it tends to limit the number of people in China may be less than 1.5 billion.

\section{Prediction}

With 2 groups of data forecast 2001 population and the 2011 population respectively.

1) With $x=2001$ substitute (8) gets $f(2001)=1276.56$ (million). Known from the 2nd set of data at the end of 2001, the actual values is 1276.27 (million), the maximum absolute error 0.29 (millions), the maximum relative error $0.023 \%$;
With $x=2011$ substitute (9), gets $f(2011)=1347.06$ (million). 2011 year's end populations which according to January, 2012 China State statistical bureau announces 1347.35 million, prediction errors is 0.29 million, its relative error $0.022 \%$.

These results indicated, when the mathematical model selection is appropriate, and then the result has certainly very high accuracy.

\section{D. $E N D$}

Promoted theory of Chebyshev optimal uniform approximation, has produced Z-Chebyshev polynomials under the $y(x)=0$ condition, while $y(x) \neq 0$ Z-Chebyshev polynomials $S_{n}(x)$ are further developing the generalized ZChebyshev $s(x)$ polynomials. For the realization of $s(x)$ polynomial, the paper gives a specific algorithm.

Apply these principles to discrete data processing in curve fitting, can establish Z-Minimax curve fitting method. This method applies to a range from "zero" began to "zero" end of description, and has a certain prediction capabilities. For a wide range of changes, sometimes it is hard to find an appropriate description, in this case, may use "the zero" to divide it certain small ranges, use "the maximum absolute error minimization" discovers the gradual change rule, again compiles these rules a total change tendency. As any segmentation, this approximation has broad applicability.

\section{REFERENCES}

[1] W. Zongde, "Two New Kinds of the Tchebycheff polynomials," Journal of Beijing University of posts and telecommunication, vol. 12, pp. 46-54, 1989.

[2] G. Lemin, "Predictive type of Chebyshev polynomials," Computer Engineering and Applications, vol. 48, pp. 34-38, 2012.

[3] G. Lemin, "Best Uniform Approximation Polynomial of Cosine Function type," Journal on Numerical Methods and Computer Applications, vol. 33, pp. 173-180, 2012.

[4] G. Lemin, "Nonlinear Chebyshev Solution Realization Question," in Proc. The 2nd International Conference on Computer Science and Service System (CSSS2012), vol. 3, pp. 1784-1789, 2012.

[5] L. M. Gu, "Chebyshev Best Approximation Theory in the Calculation of Absolute and Relative Methods of Application," 2012 International Conference on System Simulation, pp. 487-493, 2012.

[6] C. Maolin, "Parameter Estimation of Richards Model and Its Application," vo1. 40, pp. 139-143, 2010.

[7] F. J. A. Richards, "Flexible growth function for empirical use," $J$. Exp. Bot, pp. 290, 1959.

[8] China Statistical Year Book. [Online]. Available: http://www.stats.gov.cn/tjsj/ndsj/2011/indexch.htm

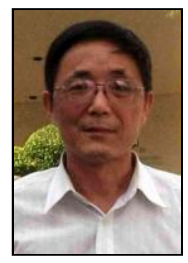

Lemin Gu is from Tongji University, Shanghai, 201804 China. $\mathrm{He}$ is an amateur math lover. His research directions are Computational mathematics; Approximation theory; Data processing, etc. 